\title{
Elevated Choroidal Thickness and Central Serous Chorioretinopathy in the Fellow Eyes of Patients with Circumscribed Choroidal Hemangioma
}

\author{
Ryan S. Kim ${ }^{a, b}$ Rishabh R. Jain ${ }^{a}$ David M. Brown ${ }^{a, f}$ Maria E. Bretana ${ }^{a}$ \\ Eric N. Kegley ${ }^{a}$ Michael A. Singer ${ }^{c}$ Antonio V. Aragon ${ }^{d, e}$ Amy C. Schefler ${ }^{a} f$ \\ ${ }^{a}$ Retina Consultants of Houston, Houston, TX, USA; ${ }^{b}$ McGovern Medical School at the University of Texas \\ Health Science Center, Houston, TX, USA; ' $M$ Medical Center Ophthalmology Associates, San Antonio, TX, USA;

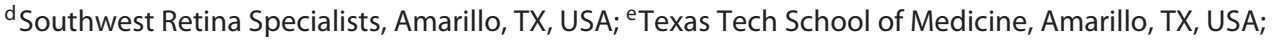 \\ fBlanton Eye Institute, Houston Methodist Hospital, Weill Cornell Medicine, Houston, TX, USA
}

\section{Keywords}

Serous chorioretinopathy · Choroidal hemangioma ·

Choroidal thickness · Contralateral eye

\begin{abstract}
Objective: To determine whether increased choroidal thickness $(\mathrm{CT})$ is associated with circumscribed choroidal hemangioma $(\mathrm{CCH})$ and whether patients with $\mathrm{CCH}$ are predisposed to central serous chorioretinopathy (CSCR). Methods: We performed a retrospective consecutive observational study of 15 fellow eyes of 15 patients who had $\mathrm{CCH}$. A- and B-scan ultrasonography, fluorescein angiography (FA), and optical coherence tomography (OCT) findings were reviewed to confirm the diagnosis of $\mathrm{CCH}$. Fellow eye $\mathrm{CT}$ was measured using enhanced depth imaging OCT. FA, indocyanine green angiography, and OCT images of fellow eyes were reviewed for signs of CSCR. Results: Mean subfoveal CT was significantly greater in the fellow eyes of patients with $\mathrm{CCH}$ than in age-matched normal eyes $(p=0.004)$. Three of $15(20.0 \%)$ fellow eyes of $\mathrm{CCH}$ patients had retinal pigment epithelium disruptions and/or pigment epithelial detachments indicative of previous asymptomatic CSCR. Conclusion: In this pilot study, elevated CT was associated with a
\end{abstract}

risk of developing $\mathrm{CCH}$ as well as CSCR in patients of varying ages. Patients diagnosed with $\mathrm{CCH}$ should be screened for CSCR in the fellow eye. Further exploration of this association may reveal useful clues about the biology of abnormally elevated choroidal hyperpermeability and its various clinical manifestations.

(c) 2018 S. Karger AG, Basel

\section{Introduction}

Circumscribed choroidal hemangiomas (CCHs) are rare, potentially congenital vascular hamartomas that typically present as an elevated mass with reddish-orange pigmentation on ophthalmoscopy. These benign, often asymptomatic tumors commonly go undiagnosed until symptoms develop possibly from subretinal fluid and macular edema $[1,2]$. Testing used to confirm the diagnosis of $\mathrm{CCH}$ includes fluorescein angiography (FA), indocyanine green angiography (ICGA), A- and B-scan

This study was performed at Retina Consultants of Houston, Houston, TX, USA.

\section{KARGER}

(c) 2018 S. Karger AG, Basel

E-Mail karger@karger.com

www.karger.com/oop
Amy C. Schefler, MD

Retina Consultants of Houston

6560 Fannin St, Suite 750

Houston, TX 77030 (USA)

E-Mail acsmd@ houstonretina.com 


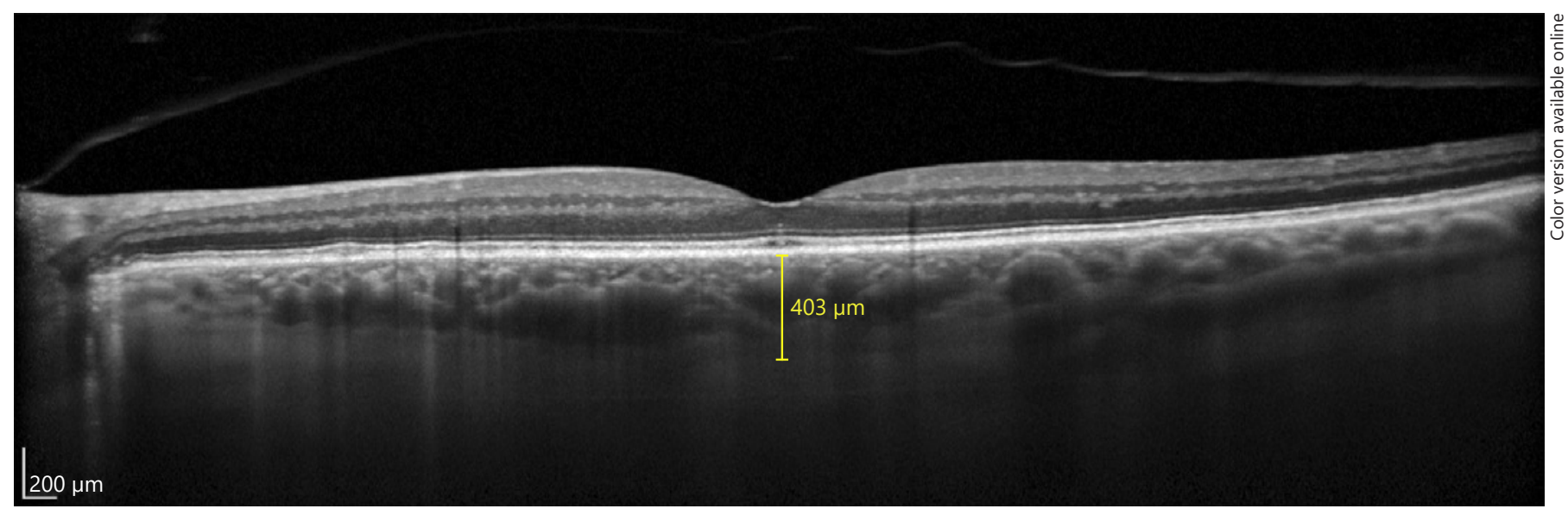

Fig. 1. Enhanced depth imaging optical coherence tomography image showing the choroid in the fellow left eye of a 74-year old circumscribed choroidal hemangioma patient. The choroid is significantly thicker than average in normal eyes. Choroidal thickness measurements were obtained using the built-in caliper in the Heidelberg Eye Explorer software. Measurements were made to the choroid stroma located at the choroid-sclera junction.

ultrasonography, and optical coherence tomography (OCT) [3]. Subretinal fluid associated with CCH typically responds well to treatment with photodynamic therapy. Photodynamic therapy has been shown to cause reduction in size of the vascular mass, resolution of subretinal fluid, decreased hyperpermeability of choroidal blood vessels, and thinning of the choroid [4].

Enhanced depth imaging OCT (EDI-OCT) has been used to produce detailed images of the choroid, and reproducible measurements of choroidal thickness (CT) have been obtained from EDI-OCTs in normal eyes [5, 6]. More recently, the method has been used to determine CT in eyes with varying types of pathology (Fig. 1) [7-9]. The objective of this study was to present evidence of an association between elevated $\mathrm{CT}$ and $\mathrm{CCH}$. Using highresolution spectral-domain OCT imaging, we also found evidence that $\mathrm{CCH}$ patients have a predisposition to central serous chorioretinopathy (CSCR). To our knowledge, this study is the first to report such an association.

\section{Methods}

This retrospective consecutive case series examined $\mathrm{CCH}$ patients evaluated at Retina Consultants of Houston (Houston, TX, USA) from December 2012 to September 2016.

\section{Participants and Sample Selection}

All CCH patients who underwent high-resolution EDI-OCT imaging were included in this study. A total of 15 patients diagnosed with $\mathrm{CCH}$ had adequate data for inclusion. The diagnosis of $\mathrm{CCH}$ was confirmed via chart review of findings from clinical ex- amination, A- and B-scan ultrasonography, FA, ICGA, and OCT. An Aviso S (Quantel Medical, Paris, France) ultrasound was used to obtain A and B scans of the lesion in the affected eye. Fundus photography was performed using an Optos 200TX (Optos, Dunfermline, UK). OCT, FA, and ICGA images were obtained using Spectralis HRA+OCT (Heidelberg Engineering, Heidelberg, Germany).

Eyes were excluded from the study if the choroidal hemangioma was diffuse or if it was associated with systemic conditions such as Sturge-Weber syndrome. Highly myopic eyes with a refractive error $>6.0$ diopters or a deviation $\geq 1 \mathrm{~mm}$ from the average axial length have been shown to have extremely thin choroids $[5,6$, $8-11]$. These eyes were neither included in the reference database of normal CT [9] nor in a pilot study by Margolis and Spaide [6]. Refractive error data were not available for all subjects. However, the refractive error was determined via chart review for all eyes with any signs of myopia including increased curvature of the retina in OCT images, and/or signs of parapapillary atrophy in B-scan ultrasonography. Axial length measurements were obtained via A-scan ultrasonography for all eyes with signs of myopia to confirm the presence of increased axial length. Two patients were excluded from our analysis because they were confirmed to be high myopes, affecting the ability to compare their $\mathrm{CT}$ measurements to historical controls. Other exclusion criteria included the presence of ocular diseases in the fellow eye, poor resolution of diagnostic images, or inability to assess the fellow eye.

Demographic data including age, sex, and race/ethnicity were recorded. Other clinical variables that were collected included subfoveal CT, signs of high myopia, and signs of CSCR. CT was defined as the distance from the posterior side of the hyperreflective band of the retinal pigment epithelium (RPE) to the choroid stro$\mathrm{ma}$ at the choroid-sclera junction. The choroid stroma was selected as the distal boundary for CT measurements based on recent evidence that this boundary provides the most reliable CT measurements [12]. Signs of CSCR were defined as serous pigment epithelial detachments or RPE/ellipsoid zone disruptions observed 

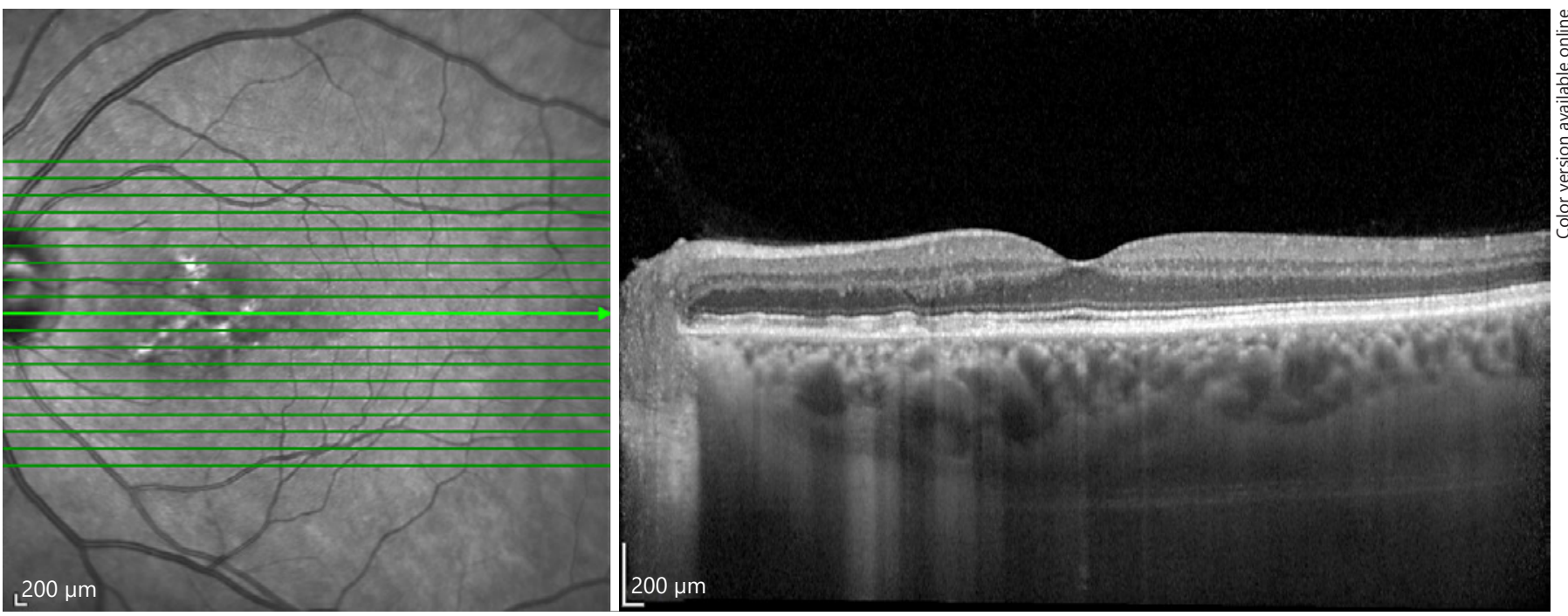

Fig. 2. Optical coherence tomography (OCT) image of the fellow eye of a 63-year-old circumscribed choroidal hemangioma patient in this study who had signs of central serous chorioretinopathy (CSCR) in the fellow eye. Note that the OCT image shows a small pigment epithelial detachment and area of retinal pigment epithelium atrophy in the fellow eye consistent with old CSCR.

on spectral-domain OCT images (Fig. 2) as well as hyperfluorescent and hypercyanescent areas on FA and ICGA images (Fig. 3) $[13,14]$.

High-resolution EDI-OCT images of the choroid in the fellow eye were obtained using Spectralis HRA+OCT based on the methods of Spaide et al. [5]. The fellow eye was chosen for this measurement so that the size of the choroidal hemangioma would not alter the measurement of the native choroidal anatomy [13]. EDI-OCT images were reviewed using the Heidelberg Eye Explorer software (Heidelberg Engineering). Measurements of CT were made under the fovea to the choroidal stroma using the integrated caliper in the Eye Explorer software (Fig. 1). Each eye was measured by three independent graders. Deviations $>15 \%$ from the mean of the three measurements were reviewed until a consensus was reached. Comparisons were made to CT data from age-matched normal patients obtained from the database published by Abbey et al. [9].

\section{Statistical Analysis}

All statistical calculations were performed using the IBM Statistical Analysis Software Package (SPSS) (IBM, Armonk, NY, USA). Mean age was calculated for $\mathrm{CCH}$ patients at the time of EDI-OCT. Mean and median CT data were calculated for the entire sample cohort as well as for each decade of life. An intraclass correlation was performed to determine interrater reliability. A one-tailed independent-samples $t$ test was performed to compare the mean subfoveal $\mathrm{CT}$ in $\mathrm{CCH}$ fellow eyes and in age-matched normal eyes. Mean subfoveal CT was calculated with respect to age, sex, race/ethnicity, and laterality of the disease. A one-way analysis of variance (ANOVA) test was performed to determine the relationship between mean CT and age group. Two-tailed, independent-samples $t$ tests were performed to determine the relationship between mean CT and sex, race, and laterality.

Choroidal Thickness in Choroidal

Hemangioma Fellow Eyes

\section{Results}

This study included 12 males $(80.0 \%)$ and 3 females $(20.0 \%)$ with a mean age of 58.1 years (median, 63; standard deviation, 16.4; range, 17-75). Eleven of the 15 patients $(73.3 \%)$ were white/non-Hispanic, 2 (13.3\%) were white/Hispanic, and the remaining 2 (13.3\%) chose not to report their race/ethnicity. Nine eyes had choroidal hemangioma in the right eye $(60.0 \%)$ and 6 had choroidal hemangioma in the left eye $(40.0 \%)$. An intraclass correlation analysis showed an interrater reliability $(\kappa)$ of 0.980 among the three graders (95\% confidence interval $0.919-$ 0.994). Mean and median CT for all patients were calculated with respect to age group, sex, and race/ethnicity. The mean fellow eye CT in the sample was $355.1 \mu \mathrm{m}$ (median, 328.3; standard deviation, 103.2; range, 226.7$550.0)$. The mean CT in the age-matched normal population was $286.0 \mu \mathrm{m}$ (Table 1). An unpaired, one-tailed $t$ test showed that the mean subfoveal CT in the CCH fellow eyes was significantly greater than that in the age-matched normal population $(p=0.004)$.

A one-way ANOVA revealed no significant relationship between subfoveal CT in CCH fellow eyes and the decade of life (age) $(p=0.307)$. Unpaired two-tailed $t$ tests were performed to determine the impact of sex and laterality on fellow eye CT. These tests revealed no significant difference in subfoveal CT between the 12 males and the 

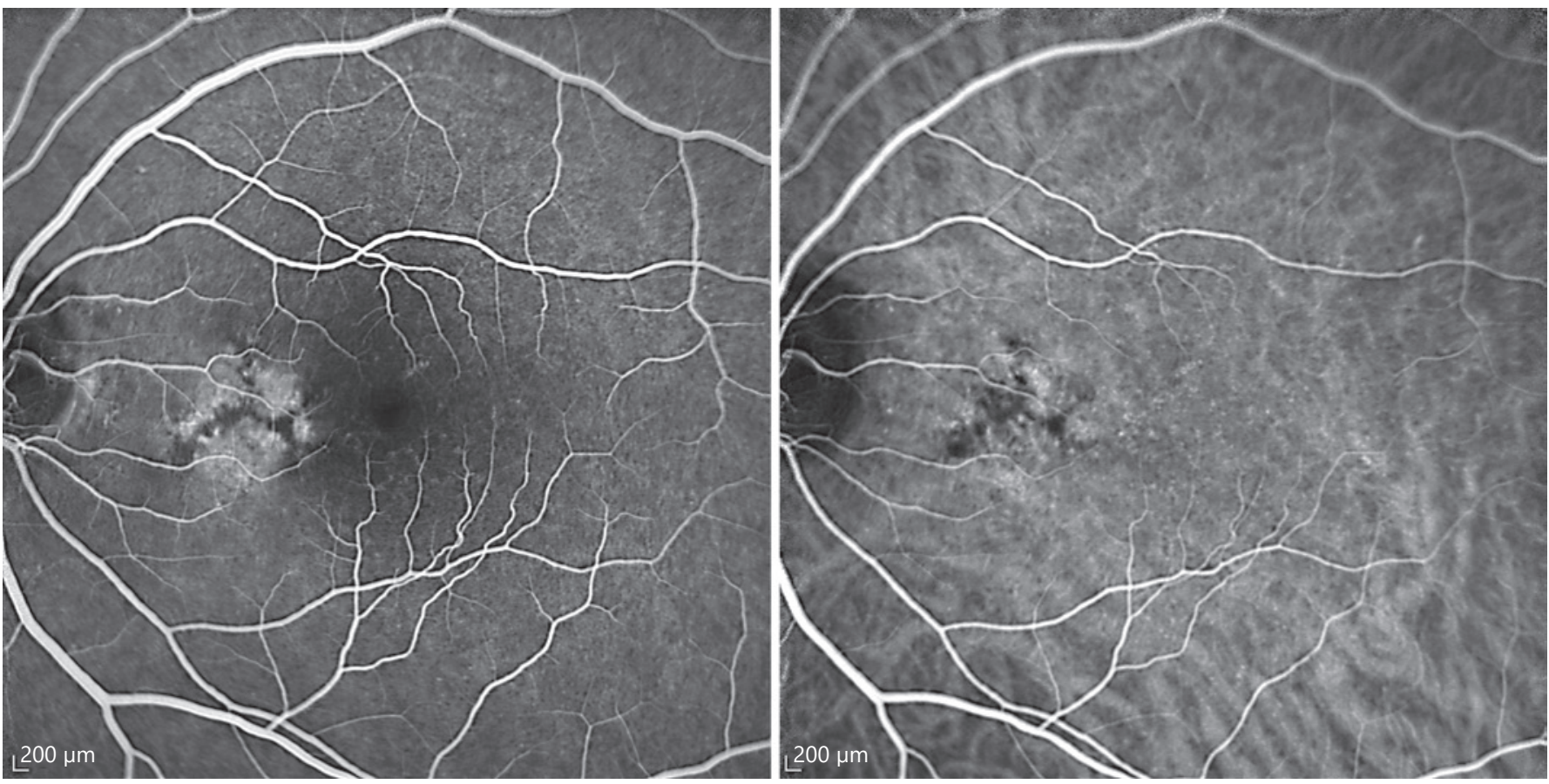

Fig. 3. Fluorescein angiography and indocyanine green angiography images of the fellow eye of the patient in Figure 2 demonstrating hyperfluorescent and hypercyanescent areas, consistent with central serous chorioretinopathy.

Table 1. Mean subfoveal CT in CCH normal and fellow eyes

\begin{tabular}{|c|c|c|c|c|c|c|}
\hline $\begin{array}{l}\text { Age, } \\
\text { years }\end{array}$ & $\begin{array}{l}\mathrm{Pa}- \\
\text { tients }\end{array}$ & $\begin{array}{l}\text { Mean CT } \\
\text { of CCH } \\
\text { fellow } \\
\text { eye, } \mu \mathrm{m}\end{array}$ & $\begin{array}{l}\text { Median } \\
\mathrm{CT} \text { of } \\
\mathrm{CCH} \\
\text { fellow } \\
\text { eye, } \mu \mathrm{m}\end{array}$ & $\begin{array}{l}\text { Age- } \\
\text { matched } \\
\text { normal } \\
\text { CT, } \mu \mathrm{m}^{\mathrm{a}}\end{array}$ & $\begin{array}{l}\text { Patients } \\
\text { with } \\
\text { signs of } \\
\text { CSCR }\end{array}$ & $\begin{array}{l}\text { Weighted } \\
\text { normal } \\
\text { mean CT, } \\
\mu \mathrm{m}^{\mathrm{b}}\end{array}$ \\
\hline $10-19$ & 1 & 234.0 & 236.0 & NA & 0 & NA \\
\hline $20-29$ & 0 & NA & NA & 339.3 & 0 & $0 \times 339.3$ \\
\hline $30-39$ & 1 & 228.3 & 223.0 & 306.6 & 0 & $1 \times 306.6$ \\
\hline $40-49$ & 1 & 226.7 & 230.0 & 342.9 & 0 & $1 \times 342.9$ \\
\hline $50-59$ & 4 & 390.8 & 396.3 & 302.7 & 0 & $4 \times 302.7$ \\
\hline $60-69$ & 4 & 412.6 & 413.5 & 243.4 & 1 & $4 \times 243.4$ \\
\hline $70-79$ & 4 & 355.8 & 367.5 & 234.3 & 2 & $4 \times 234.3$ \\
\hline $80-89$ & 0 & NA & NA & 221.8 & 0 & $0 \times 221.8$ \\
\hline otal & 15 & 355.1 & NA & 286.0 & 3 & 251.4 \\
\hline
\end{tabular}

Subfoveal CT was measured in CCH fellow eyes from the outer aspect of the RPE to the choroidal stroma. Subjects were divided into age groups coinciding with decade of life and mean CT was calculated for each age group. These values were compared with data from Abbey et al. [9]. Data were more robust in groups with three or more individuals. $\mathrm{CCH}$, circumscribed choroidal hemangioma; CT, choroidal thickness; RPE, retinal pigment epithelium. ${ }^{2}$ No CT data were available for normal eyes in the age group 10-19 years. ${ }^{b}$ Weighted mean CT in normal eyes based on the frequency of individuals in each age group in the study sample.
3 females $(p=0.837)$. Likewise, no significant difference in CT was observed between 6 right eyes and 9 left eyes $(p=0.080)$. An unpaired one-tailed $t$ test was performed to determine the impact of race/ethnicity on CT. This test revealed that fellow eye CT in 11 white/non-Hispanic eyes was significantly greater than that in 2 white/Hispanic eyes $(p=0.033)$. Two patients who chose not to report race/ethnicity were not included in this last calculation.

For $12 \mathrm{CCH}$ patients, the subfoveal CT in the fellow eye was greater than the mean CT for normal patients in the corresponding age group. RPE changes indicative of previous asymptomatic CSCR were present in 3 of 15 (20.0\%) of the fellow eyes, all of which had thick choroids compared to age-matched controls. Of note, all these 3 patients were known to be former smokers, and none of them was known to be using steroids at the time of evaluation.

\section{Discussion}

$\mathrm{CCHs}$ are rare vascular hamartomas that are often only discovered incidentally, or after secondary symptoms have developed possibly due to the presence of sub- 
foveal subretinal fluid. In this study, we used high-resolution EDI-OCT imaging to analyze the CT in the fellow eye of $\mathrm{CCH}$ patients in relation to the mean $\mathrm{CT}$ of agematched normal eyes. Spaide and colleagues $[5,6]$ and other authors have previously demonstrated the effectiveness of EDI-OCT in imaging the choroid. In addition, it has been shown that CT can be reliably measured from EDI images in normal eyes and those with different ocular pathologies [5-11, 13-19]. Abnormal CT has been previously implicated in many different inflammatory conditions such as CSCR $[7,8]$, vitelliform macular dystrophy, and other inflammatory conditions [9]. Diseases such as pachychoroid pigment epitheliopathy, CSCR, and polypoidal choroidal vasculopathy are thought to occur because of a pachychoroid-driven process involving choroidal congestion and choroidal hyperpermeability manifested by choroidal thickening and dilated choroidal vessels [20]. Of note, pachychoroid epitheliopathy syndrome and CSCR are clinically impossible to distinguish.

In this sample of 15 consecutive $\mathrm{CCH}$ patients who met the inclusion criteria for the study, mean subfoveal $\mathrm{CT}$ in the fellow eye was $355.1 \mu \mathrm{m}$. This was significantly greater than the mean CT of $286 \mu \mathrm{m}$ observed in a normal population $(p=0.004)$. In addition, we observed that the mean subfoveal CT in CCH fellow eyes of white/non-Hispanic patients was significantly greater than in $\mathrm{CCH}$ fellow eyes of white/Hispanic patients $(p=0.033)$. Further studies with larger numbers of patients are required to elucidate the relationship between race, ethnicity, and CT in this population.

In this cohort, no significant differences in CT were observed based on age $(p=0.307)$, sex $(p=0.837)$, or laterality $(p=0.080)$ of the fellow eye. Although the lack of a correlation between laterality and CT is consistent with previous findings, age and sex have been shown to be correlated with CT [17]. These CT inconsistencies in relation to age and sex may have been due to the small size of the study cohort or to the fact that eyes with elevated CT may not undergo the same age-related atrophy over time as those with normal anatomy.

We are not aware of any previous evidence of an association between CCH and CT or CSCR. It has been well documented previously that there may be a relationship between increased CT and CSCR $[7,8]$. Due to the altered choroidal anatomy in $\mathrm{CCH}$ fellow eyes, we analyzed the OCT images of fellow eyes for signs of CSCR. Although RPE disruptions can occur in other ocular pathologies, we found RPE changes highly indicative of previous asymptomatic CSCR in 3 of 15 (20.0\%) fellow eyes, a frequency far greater than expected in the normal popula-

Choroidal Thickness in Choroidal

Hemangioma Fellow Eyes tion. All of these cases were observed in patients above the age of 50 years. In addition, all three of these cases were in eyes that also exhibited elevated CT. Thus, the anatomic predisposition to increased CT, hypercongestion, and hyperpermeability in eyes with CSCR may also predispose patients to the development of $\mathrm{CCH}$.

Like all small retrospective studies, our report has some limitations. Due to the rare nature of $\mathrm{CCH}$, only 18 consecutive $\mathrm{CCH}$ patients seen initially at Retina Consultants of Houston, between December 2012 and September 2016, met the study criteria. Of these 18 patients, 2 were classified as highly myopic and subsequently excluded. These eyes are known to have very thin choroids and thus were excluded from the analysis as they have been from published normative databases of CT. In addition, only low-resolution EDI-OCT images were available for 1 patient, and therefore this patient was excluded. As a result, CT calculation was limited to a total of 15 fellow eyes. A multicenter prospective collaboration in which more $\mathrm{CCH}$ patients can be observed would be ideal.

Several authors have reported that EDI-OCT images are not as clear in patients with a thickened choroid [12, 21]. Specifically, it is more difficult to discern the distal boundary of the choroid at the choroid-sclera junction when the choroid is thickened [12]. In this study, CT was measured in the subfoveal space from the outer surface of the RPE to the proximal edge of the hyperreflective band at the choroid-sclera junction. Many of the choroids were thickened, and therefore a clear hyperreflective band was not observed in all images. As a result, there was added variability in the measurements of CT as the determination of the boundary of the choroid was not exact. In order to minimize this error, measurements were performed by three independent individuals and the mean was calculated. In order to limit variability, measurements that differed by $>15 \%$ from the mean CT were reviewed until a consensus was reached. An intraclass correlation analysis showed excellent interrater reliability between the graders $(\kappa=0.980)$.

In this study, we have demonstrated novel evidence of an association between CCH, CSCR, and increased CT. We believe that, as in previous published studies, these conditions may all be related to hyperpermeability of the blood vessels in the choroid $[8,15,22]$. Further studies are required in patients with $\mathrm{CCH}$ to determine the exact mechanism by which $\mathrm{CCH}$ and CSCR develop in this group. Given this novel association, we recommend that CSCR patients be examined for $\mathrm{CCH}$ and that $\mathrm{CCH}$ patients be examined for CSCR. 


\section{Statement of Ethics}

The protocol of this study adhered to the tenets of the Declaration of Helsinki, and institutional review board/ethics committee approval was obtained from Blanton Eye Institute, Houston Methodist Hospital (Houston, TX, USA). The study was compliant with the Health Insurance Portability and Accountability Act of 1996.

\section{Disclosure Statement}

None of the authors have any financial conflicts of interest with the material discussed in this paper. Amy C. Schefler, MD had full access to all of the data in the study and takes responsibility for the integrity and the accuracy of the data.

\section{References}

1 Shields CL, Honavar SG, Shields JA, Cater J, Demirci H: Circumscribed choroidal hemangioma: clinical manifestations and factors predictive of visual outcome in 200 consecutive cases. Ophthalmology 2001;108:22372248.

2 Karimi S, Nourinia R, Mashayekhi A: Circumscribed choroidal hemangioma. J Ophthalmic Vis Res 2015;10:320-328.

3 Berry M, Lucas LJH: Circumscribed choroidal hemangioma: a case report and literature review. J Optom 2017;10:79-83.

-4 Schmidt-Erfurth UM, Michels S, Kusserow C, Jurklies B, Augustin AJ: Photodynamic therapy for symptomatic choroidal hemangioma: visual and anatomic results. Am J Ophthalmol 2002;109:2284-2294.

-5 Spaide RF, Koizumi H, Pozonni MC: Enhanced depth imaging spectral-domain optical coherence tomography. Am J Ophthalmol 2008;146:496-500.

6 Margolis R, Spaide RF: A pilot study of enhanced depth imaging optical coherence tomography of the choroid in normal eyes. Am J Ophthalmol 2009;147:811-815.

7 Imamura Y, Takamitsu F, Margolis R, Spaide RF: Enhanced depth imaging optical coherence tomography of the choroid in central serous chorioretinopathy. Retina 2009;29: 1469-1473.

8 Mrejen S, Spaide RF: Optical coherence tomography: imaging of the choroid and beyond. Surv Ophthalmol 2013;58:387-429. $\checkmark$ Abbey AM, Kuriyan AE, Modi YS, Thorell MR, Nunes RP, Goldhardt R, Yehoshua Z, Gregori G, Feuer W, Rosenfeld PJ: Optical coherence tomography measurements of choroidal thickness in healthy eyes: correlation with age and axial length. Ophthalmic Surg Lasers Imaging Retina 2015;46:18-24.

10 Flores-Moreno I, Lugo F, Duker JS, RuizMoreno JM: The relationship between axial length and choroidal thickness in eyes with high myopia. Am J Ophthalmol 2013;155: 314-319.

11 Fujiwara T, Imamura Y, Margolis R, Slakter JS, Sapide RF: Enhanced depth imaging optical coherence tomography of the choroid in highly myopic eyes. Am J Ophthalmol 2009; 148:445-450.

12 Vuong VS, Moisseiev E, Cunefare D, Farsiu S, Moshiri A, Yiu G: Repeatability of choroidal thickness measurements on enhanced depth imaging optical coherence tomography using different posterior boundaries. Am J Ophthalmol 2016;169:104-112.

13 Roisman L, Lavinsky D, Magalhaes F, Aggio FB, Moraes N, Cardillo JA, Farah ME: Fundus autofluorescence and spectral domain OCT in central serous chorioretinopathy. J Ophthalmol 2011;2011:706849.

14 Montero JA, Ruiz-Montero JM: Optical coherence tomography characterization of idiopathic central serous chorioretinopathy. Br J Ophthalmol 2005;89:562-564.

15 Kim YK, Ryoo NK, Woo SJ, Park KH: Choroidal thickness changes after photodynamic therapy and recurrence of chronic central serous chorioretinopathy. Am J Ophthalmol 2015;160:72-84.
16 Kim YT, Kang SW, Bai KH: Choroidal thickness in both eyes of patients with unilaterally active central serous chorioretinopathy. Eye 2011;25:1635-1640.

17 Bafiq R, Mathew R, Pearce E, Abdel-Hey A, Richardson M, Bailey T, Sivaprasad S: Age, sex, and ethnic variations in inner and outer retinal and choroidal thickness on spectraldomain optical coherence tomography. Am J Ophthalmol 2015;160:1034-1043.

18 Rhodes LA, Huisingh C, Johnstone J, Fazio MA, Smith B, Wang L, Clark M, Downs JC, Owsley C, Girard MJA, Mari JM, Girkin CA: Peripapillary choroidal thickness variation with age and race in normal eyes. Invest Ophthalmol Vis Sci 2015;56:1872-1879.

19 Tuncer I, Karahan E, Zengin MO, Atalay E, Polat N: Choroidal thickness in relation to sex, age, refractive error, and axial length in healthy Turkish subjects. Int Ophthalmol 2015;35:403-410.

20 Pang CE, Freund KB: Pachychoroid neovasculopathy. Retina 2015;35:1-9.

21 Cho AR, Choi YJ, Kim YT; Medscape: Influence of choroidal thickness on subfoveal choroidal thickness measurement repeatability using enhanced depth imaging optical coherence tomography. Eye (Lond) 2014;28:11511160.

22 Jirarattanasopa P, Ooto S, Tsujikawa A, Yamashiro K, Hangai M, Hirata M, Matsumoto A, Yoshimura N: Assessment of macular choroidal thickness by optical coherence tomography and angiographic changes in central serous chorioretinopathy. Ophthalmology 2012;119:1666-1678. 